\title{
Facial Asymmetry Correction in Facial Palsy Patients with Silhouette Sutures
}

\author{
María Luisa Navarrete ${ }^{1}$, Ricard Palao ${ }^{2}$, Lluisa Torrent ${ }^{3}$, Juan Fernando Fuentes ${ }^{1}$, Mireia González ${ }^{1}$ \\ ${ }^{1}$ Department of Otorhinolaryngology, Vall d’Hebron Hospital, Autonomous University of Barcelona, Barcelona, Spain; ${ }^{2}$ Department \\ of Plastic Surgery, Vall d'Hebron Hospital, Autonomous University of Barcelona, Barcelona, Spain; ${ }^{3}$ Department of Rehabilitation, \\ Vall d'Hebron Hospital, Autonomous University of Barcelona, Barcelona, Spain. \\ Email: mlna@telefonica.net
}

Received October $4^{\text {th }}, 2011$; revised November $6^{\text {th }}, 2011$; accepted December $19^{\text {th }}, 2011$

\begin{abstract}
Introduction: over the last few decades several techniques static and dynamics, have been performed to improve facial asymmetry and functionality alter suffering facial paralysis. Methods: we present a pilot study to show and evaluate the benefits of a new form of facial suspension, with Silhouette sutures. We performed two patients with total and complete facial palsy due to otical tuberculosis in one case and to parotid carcinoma in the other. Results: one year after surgery, both patients have improved facial asymmetry, mastication and speech production which have lead to a higher self-esteem and major social interaction. Conclusions: static facial suspension with Silhouette sutures is a non invasive alternative to dynamic techniques in patients who don't want or can't undergo more complex surgeries.
\end{abstract}

Keywords: Facial Paralysis; Suture Suspension; Static Technique; Silhouette Sutures

\section{Introduction}

Facial paralysis has significant functional and aesthetic impact.

Identifying new techniques to correct facial defects with minimal side effects is gaining importance. Society places great emphasis on facial appearance and expression. In addition, severe facial paralysis hinders mastication, eye closure and speech production.

As a result, numerous reanimation techniques have been popularized to correct this problem. These methods are broadly classified into dynamic and static procedures. These procedures have their limitations. Literature revisions show no better results achieved with one or other techniques. Realistic expectations must be established between the physician and the patient, and the appropriated technique must be chosen depending on the circumstances.

Facial static suspension with Silhouette sutures enhance facial symmetry and aesthetic results as well as improve functionality, specially in the areas of mastication and speech production in the patients with facial palsy.

Thanks to the sutures that are tunnelled just below the dermis, is possible to lift the affected hemiface.

The new generation of Silhouette sutures for tissue suspension is different from the golden and russian threads, which have showed poor result. Silhouette su- tures bring a new form of anchorage by a new form of locking cones done of polypropylene and absorbable polilactic and glycolic acids (Figure 1). This suture allows tissue growth in and around the cones, and therefore a stronger anchor. Its pulling capacity has been demonstrated to be ten times stronger than that of the other sutures. Furthermore, these sutures don't migrate into other neighbour tissues as other sutures do. Also no tissues

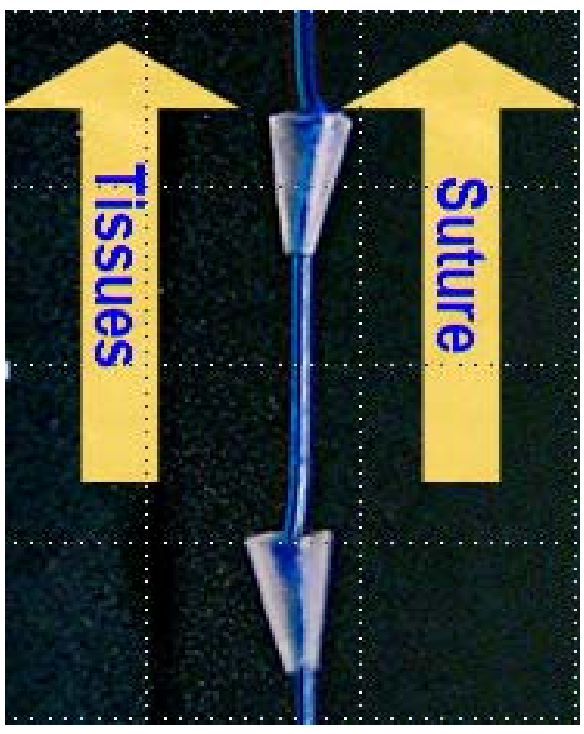

Figure 1. View of Silhouette sutures. 
injury takes place during the dissection and tunnelling of these sutures.

Approved by the FDA in November 2006 and the EEC in March 2007, their have been performed more than 6 thousand midface procedures around USA and Europe, using this kind of suture for facial cosmetics. Actually they have begun to be used to treat facial palsy [1]. Unfortunately, effectiveness of this kind of sutures, used in cosmetics have a lifetime of six to eight years. However, authors think that when using it to improve facial asymmetry after facial palsy, the effect will last longer, because of the flaccidity and absence of muscular movements in the areas suspended with the Silhouette sutures.

\section{Cases Report}

We evaluate the effectiveness of facial suspension with Silhouette sutures in two patients and describe the technique used.

Case one is a 79 year old female patient received in our Department in August 2006 because of facial palsy and left othorrea. No improvement after taking common antimicrobials took place and therefore a special bacteriological study and a CT scan was indicated. The bacteriological results showed otic tuberculosis and the CT scan a osteomyelitis of the petrous bone. Facial nerve innervation didn't take place after completing TBC treatment, therefore static and dynamic techniques where offered to the patient. Given the context of the patient, static facial procedure with Silhouette suture was considered as the best technique for this patient.

Case two is a 76-year old female patient, received in our Department April 2006 with a painless slowly growing mass in the region of the left parotid gland. The CT scan showed a deep lobe parotid tumor of $3 \times 3,5 \times 4 \mathrm{~cm}$ with central necrosis and no regional lymph node involvement. Fine-needle aspiration biopsy and cytological examination reported acinous cell carcinoma. Total parotidectomy was performed and complete facial paralysis occurred as an early complication. After declining other procedures to re-establish mimetic control of the face, the patient decided together with the physician for the new static procedure wit Silhouette sutures. Te procedure was performed, as well as the other patient, three years after facial palsy's onset.

\section{Results}

One year after surgery, both functional and cosmetic results are satisfactory. The patients, with good symmetry in repose, report functional improvement, particularly in the areas of mastication and articulation.

The static face procedures with Silhouette sutures are performed as described (Figures 2 and 3). The patient must be marked in the upright sitting position. It begins with a

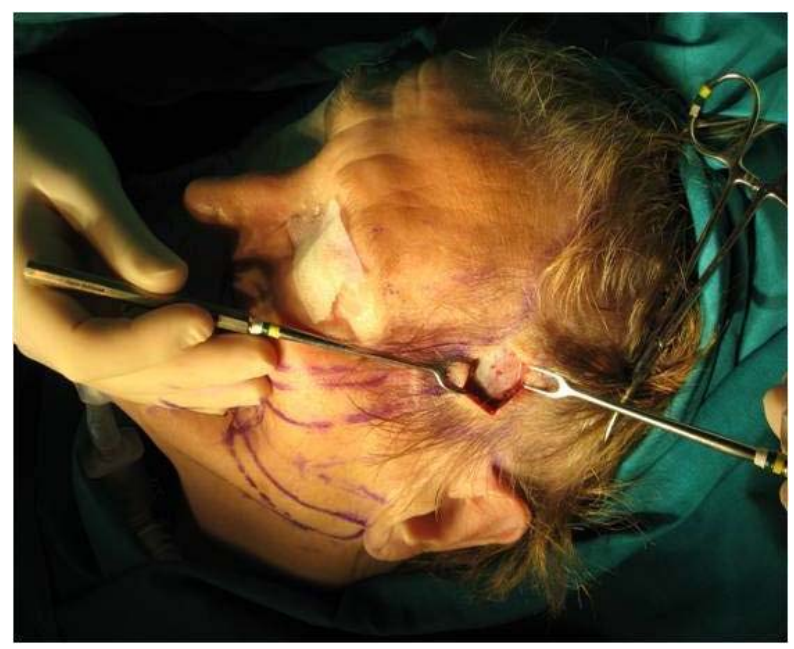

Figure 2. Approach by a small $2.5-3 \mathrm{~cm}$ incision placed in the temporal area.

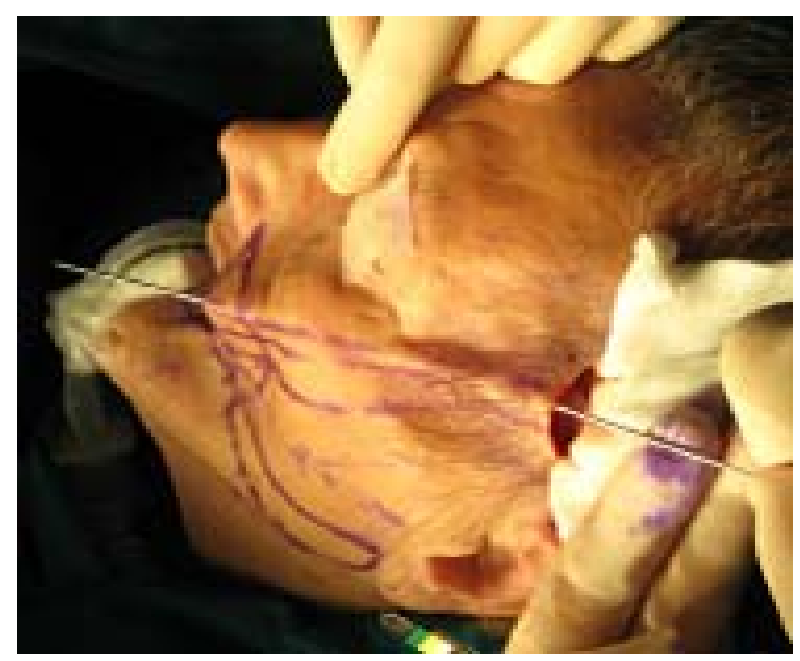

Figure 3. Dissection is used to start a plane just below the dermis.

small, $2.5-3 \mathrm{~cm}$ incision placed in the temporal area, which is covered afterward with the hair. Sharp dissecttion is used to start a plane just below the dermis, and the suture is tunnelled in the avascular facial (temporal, malar and perioral) plane. The sutures are fixed to the temporal fascia, which has been previously strengthen with a non absorbable mesh.

The patient is given the option of local anesthesia with intravenous sedation or general anesthesia. The procedure has lasted 45 minutes of average. Afterwards patient and family are informed of post-operative cares, which are similar of those recommended after facelift.

Outcome was evaluated by photodocumentation and clinical examination, one month and six months after surgery.

Both patients improved facial symmetry in repose and an elevation of the oral commissure was seen. Further- 
more both women reported that they were able to chew on the paralysed side, which was impossible before surgery. Speech production had improved as well.

Finally the patients refer an increased degree of satisfaction (Figures 4 and 5).

\section{Discussion}

Several conditions can cause facial paralysis. The severity of facial palsy depends on the underlying cause and the portion of the facial nerve that is involved. In this study we focus on complete and total paralysis, with maximum degree of degeneration, those without a reasonable likelihood of spontaneous return of function.

Facial reconstructive surgery is divided into static and dynamic procedures. Static reanimation techniques provide cosmetic and functional results and correct regional deformities as natural as possible. Dynamic techniques, used to restore function and mimetic control of the face, such as smiling [2], are broadly classified into 2 types, neural methods and musculofascial transpositions. De-

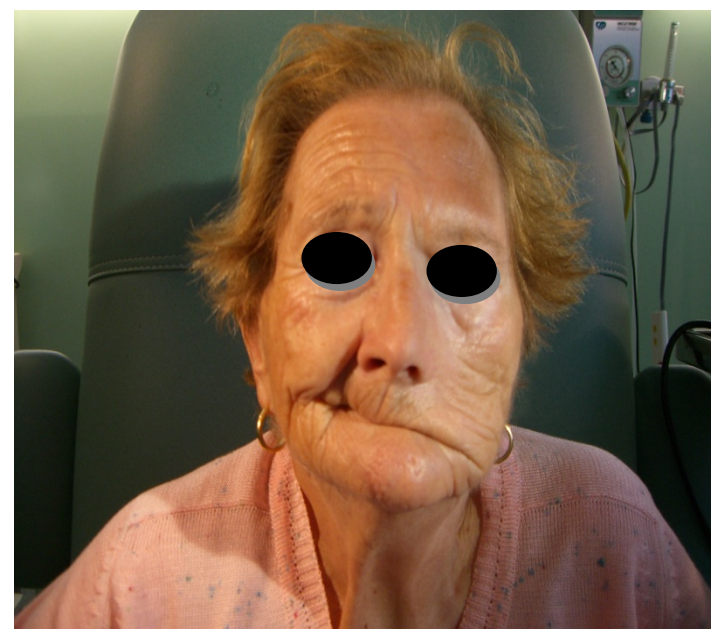

pending on the duration of the paralysis and the chance of recovery, one or the other technique is performed.

The neural methods are indicated when nerve injury or total irreversible function loss has taken place, but distal neural tubes are competent and facial muscles are yet not atrophic (less than 2 years). Neural methods are divided into 3 types: 1) Ipsilateral facial nerve anastomosis: indicated in patients with injury of isolated branches. But in case of trunk section, this technique is contraindicated because of the high risk of synkinesias; 2) Crossover facial nerve anastomosis: this procedure allows synchronized, voluntary and symmetric movements. The disadvantages of this technique are the long time needed after surgery for movement recuperation and complications on the healthy side [3]; 3) Neural transposition: is used when nerve anastomosis can't be performed. Hypoglossal nerve or another nerve of the same side can be used, with the disadvantage of using a healthy nerve and resulting an uncoordinated movement compared with the opposite face side.

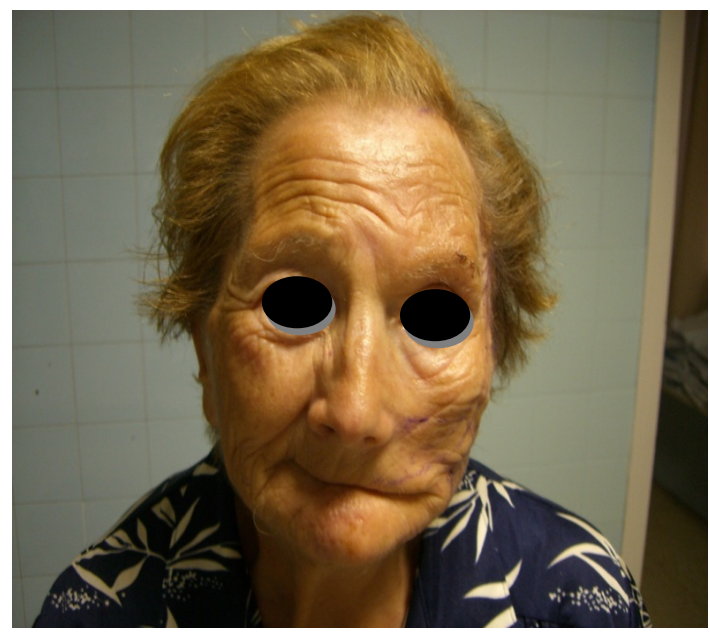

Figure 4. First patient before and after operation respectivelly.
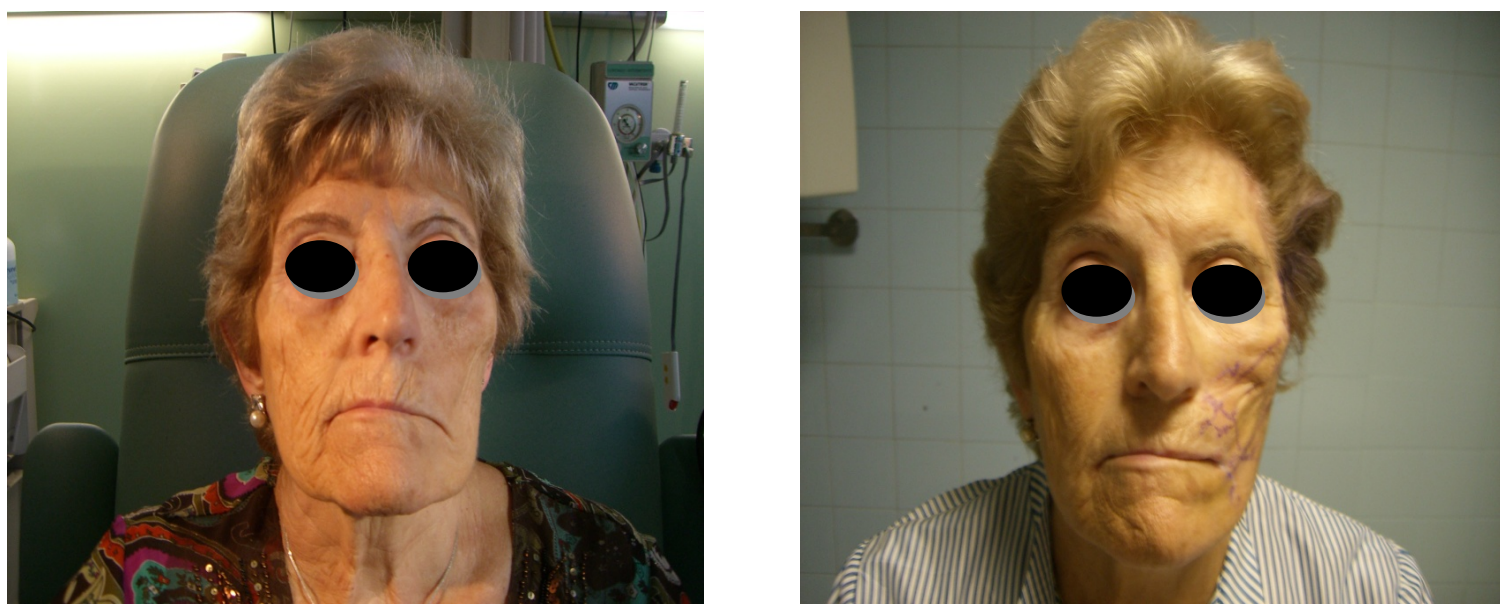

Figure 5. Second patient before and after operation respectivelly. 
Each facial nerve innervates 17 muscles responsible for facial symmetry, muscular tone and voluntary and involuntary face movements. In an ideal way, attending to fisiopatological principles, the correct movement of these muscles can only be achieved by neuro-techniques. Unfortunately, when facial palsy's onset is two or more years ago, due to neural distal tubes fibrosis and loss of motor plates, neuro-techniques are not useful anymore. In these cases, muscular transposition can be interesting. This technique is performed, particularly for lower midface reanimation [4]. Different types of muscular transposition techniques exist: 1) Temporal and masseter muscles are mostly used for local muscular transposition. Although this technique isn't able to restore emotional movement perfectly, it's a better choice than distance transpositions and neurorafia or neural grafts. Postoperative rehabilitation is of outmost importance to achieve good results. According to the oral commissure, the movement achieved by a temporal muscle transposition is on average $1 \mathrm{~cm}$, while using a free muscular transplant this drag is up to $2.5 \mathrm{~cm}$. Temporal muscle transposition is recommended when the aim is to achieve a better eye closure, by activation of the eyelid closure. Most authors have abandoned the use of masseter muscle transposition; 2) Distal or free muscular transpositions: this is considered nowadays the gold standard for patients with a long time facial palsy (onset over two years). Its long surgery time and complexity has to be taken into consideration. The muscular donor chosen must offer similar movements than the opposite face side. The gracilis muscle is a good choice due to its long neurovascular pedicle and correct action range after inervation. The disadvantage is mainly its thickness, which requires to be remodelated afterwards. Overmore the gracilis muscle has only one action vector, that's the reason why this muscle is used only to achieve some specific kind of smile.

Static procedures improve facial symmetry in repose and changes overall facial appearance. This technique can be performed to patients that are considered too old to be submitted to a dynamic procedure (over 60 years), and to patients that have been submitted to a muscular transposition [1,2]. These techniques include frontal and facial lift, malar rise, static traction strip (tendon grafts, autologous or lyophilized fascia lata $\cdots$ ). Other techniques include neurectomy, myotomies or botulinum toxin in- jection in the paralysed side when the patient suffers from synkinesis, or in the healthy side to reduce move- ment and obtain major symmetry [5,6]. All techniques have advantages and disadvantages and none of them provides optimal recovery, therefore we present a non invasive procedure, with good results and not aggressive for the patient. The sutures, tunneled under the skin raise the low part of the face. The Silhouette sutures differ from the golden sutures, not used anymore, and the $\mathrm{r} \quad \mathrm{u} \quad \mathrm{s}$ sian sutures, which have showed poor results. Silhouette sutures have some qualities: it's a safe and effective mechanism to elevate tissues, whereas sutures with tabs can damage tissues. It's a fast procedure, which takes on average 45 minutes and can be performed with local anesthesia, with a short postoperative time, normally less than 3 days. The results, when used for facial cosmetic surgery, last more or less 6 years, whereas authors think that when used in facial palsy, the results will last longer due to muscle paralysis. The absorbable cones have two advantages. On the one hand its pulling capacity has demonstrated to be ten times stronger than that of other sutures. On the other hand, the cones stay fixed under the skin and don't migrate as other sutures do. A collagen structure grows around the cones, and once the cone is reabsorbed, the collagen fibers keep pulling the tissues.

The Silhouette lift is indicated in patients with late facial palsy, when other techniques such as neural-procedures or muscular transpositions are not indicated. It can be performed as well as a complementary procedure to other more complex techniques, dynamic as well as static procedures, when results are unsatisfactory. It's recommended to tunnel at least 5 sutures under the skin. In some cases with major defects, one or two more sutures can be tunneled. Compared to other techniques, specially neural-surgery, results with Silhouette sutures can be seen soon after surgery. Definitive correction of asymmetry has been reported 2 to 3 weeks after suture implantation.

Finally, the successful management of facial palsy depends on the skillful application of a specific therapeutic modality when appropriate. Procedures with static facial suspension improve facial symmetry in repose, as well as some functional aspects in the areas of speech production and mastication in patients with complete facial paralysis. This technique offers some advantages compared to other procedures. It's a non-invasive procedure, which improves facial symmetry and some functional aspects with minimal risk and a good and short postoperative time. These findings further support the use of Silhouette sutures where possible, although more studies are required to evaluate long time results.

\section{REFERENCES}

[1] G. M. Gamboa and L. O. Vasconez, "Suture Suspension Technique for Midface and Neck Rejuvenation,” Annals of Plastic Surgery, Vol. 62, No. 5, 2009, pp. 478-481. doi:10.1097/SAP.0b013e31818c4b45

[2] D. D. Leventhal and E. A. Pribitkin, "Static Facial Suspension with Surgisis ES (Enhanced Strength) Sling," The Laryngoscope, Vol. 118, No. 1, 2008, pp. 20-23. doi:10.1097/MLG.0b013e31814b8d83 
[3] U. G. Salimbeni, "Evaluation of Results in 36 Cases of Facial Palsy Treated with Nerve Grafts," Annals of Plastic Surgery, Vol. 33, 1982, pp. 195-199.

[4] R. T. Manktelow, "Free Muscle Transplantation for Facial Paralysis," Clinics in Plastic Surgery, Vol. 11, No. 3, 1984, pp. 215-219.
[5] M. D. Wells and R. T. Manktelow, "Surgical Management of Facial Palsy," Clinics in Plastic Surgery, Vol. 17, No. 5, 1990, pp. 645-648.

[6] W. F. Larrabee, J. R. Thomas and M. Shindo, "Rehabilitation of Facial Paralysis,” Clinics in Plastic Surgery, Vol. 5, No. 2, 1997, pp. 213-216. 